\title{
A highly magnetized twin-jet base pinpoints a supermassive black hole ${ }^{\star}$
}

\author{
A.-K. Baczko1,2,3, R. Schulz ${ }^{2,1,11}$, M. Kadler², E. Ros ${ }^{3,4,5}$, M. Perucho ${ }^{5,4}$, T. P. Krichbaum ${ }^{3}$, M. Böck ${ }^{3}$, M. Bremer ${ }^{6}$, \\ C. Grossberger ${ }^{7,2,1}$, M. Lindqvist ${ }^{8}$, A. P. Lobanov ${ }^{3,9}$, K. Mannheim² ${ }^{2}$, I. Martí-Vidal ${ }^{8}$, C. Müller ${ }^{10,2,1}$, \\ J. Wilms ${ }^{1}$, and J. A. Zensus ${ }^{3}$
}

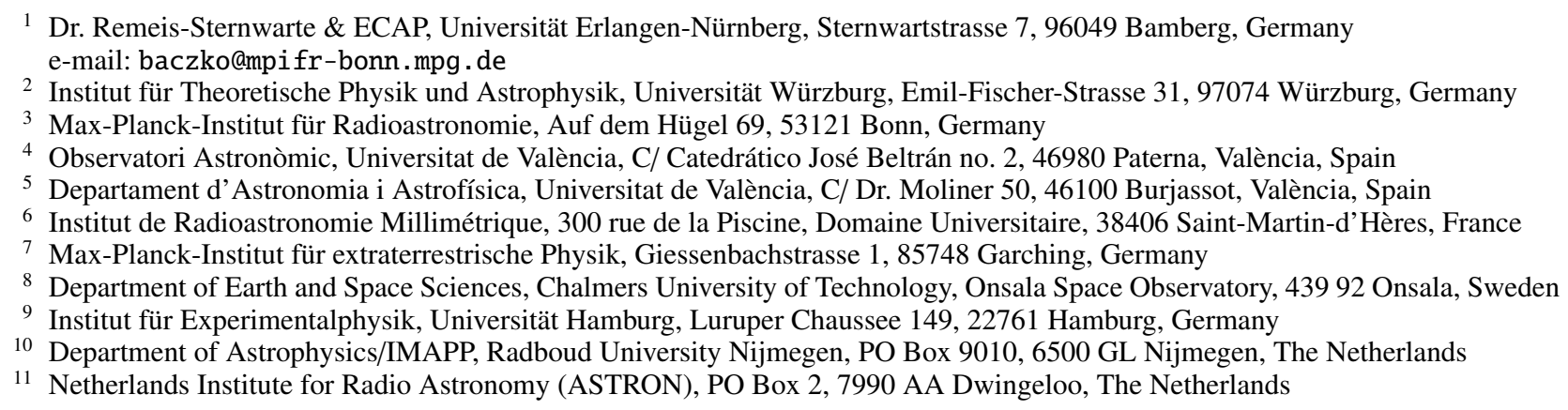

Received 14 December 2015 / Accepted 23 May 2016

\begin{abstract}
Supermassive black holes (SMBH) are essential for the production of jets in radio-loud active galactic nuclei (AGN). Theoretical models based on (Blandford \& Znajek 1977, MNRAS, 179, 433) extract the rotational energy from a Kerr black hole, which could be the case for NGC 1052, to launch these jets. This requires magnetic fields on the order of $10^{3} \mathrm{G}$ to $10^{4} \mathrm{G}$. We imaged the vicinity of the SMBH of the AGN NGC 1052 with the Global Millimetre VLBI Array and found a bright and compact central feature that is smaller than 1.9 light days (100 Schwarzschild radii) in radius. Interpreting this as a blend of the unresolved jet bases, we derive the magnetic field at 1 Schwarzschild radius to lie between $200 \mathrm{G}$ and $\sim 8.3 \times 10^{4} \mathrm{G}$ consistent with Blandford \& Znajek models.
\end{abstract}

Key words. magnetic fields - galaxies: active - galaxies: jets - galaxies: magnetic fields - galaxies: nuclei

\section{Introduction}

Together with the existence of a central supermassive black hole $(\mathrm{SMBH})$ surrounded by a circumnuclear torus, the unification scheme of radio loud active galactic nuclei (AGN; Antonucci 1993; Fanidakis et al. 2011) postulates the occurrence of two symmetric radio jets, which emanate parallel and antiparallel to the angular momentum vector of the black hole. Despite our expectations, we mostly find jets appear one-sided in radio flux density-limited samples of AGN such as MOJAVE (Lister et al. 2009). This is a selection effect due to the strongly Dopplerboosted emission of the relativistically moving plasma in those jets, which are pointed at small angles to the line of sight toward the observer (Lister \& Marscher 1997). This orientation bias poses a problem when trying to locate the putative black hole at the true center of activity because of the unknown offset between the black hole and base of the jet above the accretion disk. Following Blandford \& Znajek (1977) it is possible to launch the jets by extracting rotational energy from a Kerr black

\footnotetext{
* The VLBI images shown in Figs. 3 and 4 are only available at the CDS via anonymous ftp to cdsarc.u-strasbg. fr (130.79.128.5) or via

http://cdsarc.u-strasbg.fr/viz-bin/qcat?J/A+A/593/A47
}

hole. To test whether high magnetic fields as needed for this approach are observable, a precise knowledge of the location of the central engine is required. Ultrahigh-resolution, radiointerferometric observations with Very Long Baseline Interferometry (VLBI) have the potential to locate regions in jets with microarcsecond precision (Zensus 1997).

The core shift effect has been studied in detail in the low-luminosity AGN NGC 1052 (RA 02h41m04.8s, Dec-08d $\left.15^{\prime} 21^{\prime \prime}\right)^{1}$ at $\mathrm{cm}$ wavelengths by Kadler et al. (2004b). The source hosts a supermassive black hole with a mass of $\sim 10^{8.2} M_{\odot}$ (Woo \& Urry 2002) and a twin-jet system. It is located at a distance of $19.5 \mathrm{Mpc}^{1}$. A two-sided radio structure stretches across the inner $\sim 3 \mathrm{kpc}$ of the galaxy, interacting strongly with the interstellar medium (Wrobel 1984; Kadler et al. 2004b), from which a total jet kinetic power of $5 \times 10^{41} \mathrm{erg} \mathrm{s}^{-1}$ is estimated (Kadler et al. 2003). The parsecscale structure of the twin-jet system has been studied extensively at $\mathrm{cm}$ wavelengths using multifrequency VLBI observations (Kameno et al. 2001; Vermeulen et al. 2003; Kadler et al. 2004a), revealing a prominent emission gap between the two jets, which has been interpreted as resulting from free-free

1 NASA/IPAC Extragalactic Database (NED) and references therein. 
Table 1. Participating GMVA stations.

\begin{tabular}{|c|c|c|c|}
\hline Station & $\begin{array}{c}\text { Diameter } \\
{[\mathrm{m}]}\end{array}$ & $\begin{array}{c}\text { SEFD } \\
{[\mathrm{Jy}]}\end{array}$ & Observation \\
\hline Metsähovi & 14 & 17650 & $\checkmark$ \\
\hline Onsala & 20 & 5100 & $-^{a}$ \\
\hline Effelsberg & 100 & 930 & $\checkmark$ \\
\hline Plateau de Bure & $35^{b}$ & 450 & $\checkmark$ \\
\hline Pico Veleta & 30 & 640 & $\checkmark$ \\
\hline VLBA NL & 25 & 4550 & $\checkmark$ \\
\hline VLBA FD & 25 & 3030 & $\checkmark$ \\
\hline VLBA LA & 25 & 1390 & $\checkmark$ \\
\hline VLBA KP & 25 & 3450 & $\checkmark$ \\
\hline VLBA PT & 25 & 2270 & $\checkmark$ \\
\hline VLBA OV & 25 & 3570 & $\checkmark$ \\
\hline VLBA BR & 25 & 3230 & $\checkmark$ \\
\hline VLBA MK & 25 & 5560 & $\checkmark$ \\
\hline
\end{tabular}

Notes. ${ }^{(a)}$ Data loss due to receiver problems. ${ }^{(b)}$ Equivalent $(5 \times 15 \mathrm{~m})$.

absorption in a circumnuclear torus with a column density of $N_{\mathrm{H}} \sim 10^{22}-10^{24} \mathrm{~cm}^{-2}$ (Kameno et al. 2001; Vermeulen et al. 2003; Kadler et al. 2004a,b; Brenneman et al. 2009) around a probably rapidly rotating black hole (Brenneman et al. 2009).

The free-free absorption effects that dominate the morphology of the source at $\mathrm{cm}$ wavelengths (Kameno et al. 2001; Vermeulen et al. 2003; Kadler et al. 2004a) are negligible at $3 \mathrm{~mm}$ (corresponding to a frequency of $86 \mathrm{GHz}$ ). Therefore, observations at $86 \mathrm{GHz}$ are able to peer through the absorbing structure and reveal insights into the jet-launching region of NGC 1052, an exceptional case of a double-sided jet system.

In this work, we present results from one observation in 2004 of the twin-jet system of NGC 1052 at $86 \mathrm{GHz}$ with the Global $\mathrm{mm}-\mathrm{VLBI}$ Array $\left(\mathrm{GMVA}^{2}\right)$, yielding an unobscured view of the innermost structures in the twin-jet system, where 1 mas corresponds to a projected linear size of $0.094 \mathrm{pc}$.

It is a challenge to observe weak and low-declination sources such as NGC 1052 with VLBI observations at such a high frequency, especially in terms of sensitivity. Present GMVA capabilities offer a large number of telescopes capable of observing at $86 \mathrm{GHz}$ and much enhanced data bit rates, which lead to a high enough visibility sampling to image the twin-jet system with the highest resolution to date. We discuss the data reduction in the next section and report the results and conclusions from our successful calibration and imaging in Sects. 3 to 5.

\section{Observation and data reduction}

The observations were performed during the Session II (October) of the GMVA in 2004 in experiment GK027, from October 09 to 10 at a frequency of $86 \mathrm{GHz}$ with 15 antennas (see Table 1). The European antennas started on October 9 at 22:00 UT and ended at 07:00 UT, whereas the US array observed from 02:00 to 14:00 UT. The correlator accumulation time was $1 \mathrm{~s}$, the data were recorded in setup mode 512-8-2, namely, with eight $16-\mathrm{MHz}$ channels in left-hand circular polarization. The observing cycle was several blocks of $20 \mathrm{~min}$, consisting in one 1-min scan on the calibrator $0235+164,2$ min for

\footnotetext{
2 http://www3.mpifr-bonn.mpg.de/div/vlbi/globalmm/
}

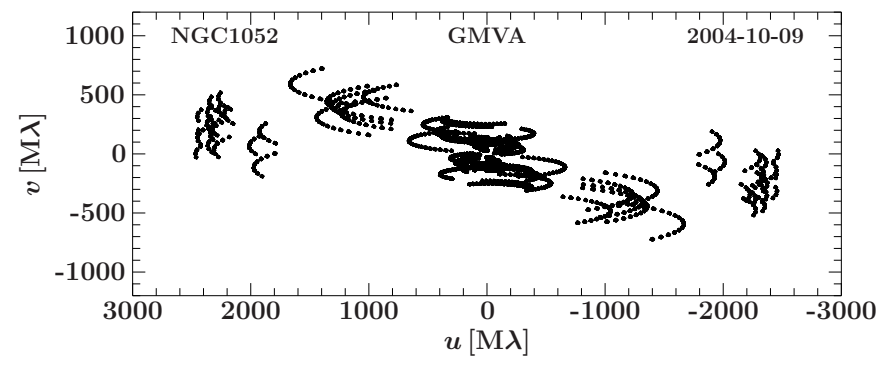

Fig. 1. $(u, v)$-coverage of NGC 1052 at $86 \mathrm{GHz}$ with the GMVA.

slewing, by $3 \mathrm{~m} 24 \mathrm{~s}$ on objectNGC 1052, and $13.5 \mathrm{~min}$ for pointing and calibration and slewing back to $0235+164$. $0716+714$ was observed as calibrator immediately prior to the NGC 1052 block, and OJ 287 was observed at the end. Data were recorded with the Mark IV and Mark V systems and correlated at the processing center at the Max-Planck-Institut für Radioastronomie, Germany. The final $(u, v)$-coverage is shown in Fig. 1 and reaches maximum values of $\sim 2.2 \mathrm{G} \lambda$ in the east-west direction.

Amplitude and phase calibration were performed using the NRAO Astronomical Image Processing System (AIPS). The phase calibration was performed in two steps after correcting for changes in the parallactic angle. First, the sub-band phase calibration corrects for station dependent delays and phase offsets with each sub-band generated from differences in the electronic systems of each station (Martí-Vidal et al. 2012a). In contrast to a manual phase and delay calibration we used an interpolated smoothed solution to align the phases of all sub-bands, also referred to as intermediate frequencies (IFs). This solution was found by interpolating the subset of best phase and delay solutions for each individual IF referred to as one fiducial IF. The multiband fringe fitting corrects for the multiband antennarelated gains with the use of global fringe fitting. For the fringe search, we used the AIPS task FRING. We selected data above a signal-to-noise ( $\mathrm{S} / \mathrm{N})$ threshold of 4.5 (aparm(7) in FRING). The search windows were of $20 \mathrm{~ns}$ and $100 \mathrm{mHz}$ for delay and delay rate, respectively (parameters dparm(2) and dparm(3), respectively). After several tests, we found that the highest number of successful detections was obtained with an integration time of $1 \mathrm{~min}$ (solint parameter in FRING). This is a good trade-off between phase degradation due to coherence times of 20-30 s and detection as a function of the square root of the integration time. We used Pico Veleta and Los Alamos as reference antennas and we applied an exhaustive fringe search $(\operatorname{aparm}(9)=1)$ first to the Effelsberg and then to the Pie Town stations. Delay and rate solutions with a very clear departure from the average solutions for each antenna were flagged after visual inspection. The amplitude calibration was made using weather data recorded at each station and measurements of the system temperatures at all observing telescopes. We used the task APCAL. After calibration, the phases are flat and the amplitude can be expressed in units of jansky (prior to calibration those are simply correlation coefficients).

After the calibration steps in AIPS, hybrid mapping with the DIFMAP software (Shepherd et al. 1994) was performed. The data were rebinned to $10 \mathrm{~s}$, and statistical errors were determined from the scatter within these bin periods for each baseline. The visibilities were carefully inspected and bad data points were flagged. After building a first basic model using the CLEAN algorithm implemented in DIFMAP and phase self-calibration, the data were edited again. Once a good model was found, the visibilities were amplitude self-calibrated over the complete 
Table 2. Image parameters of the GMVA observation from 2004-10-09.

\begin{tabular}{lccccccc}
\hline \hline Weighting & $\begin{array}{c}\text { Taper } \\
{[\mathrm{M} \lambda]}\end{array}$ & $\begin{array}{c}S_{\text {total }} \\
{[\mathrm{mJy}]}\end{array}$ & $\begin{array}{c}S_{\text {peak }} \\
{[\mathrm{mJy} / \mathrm{beam}]}\end{array}$ & $\begin{array}{c}\sigma_{\text {RMS }} \\
{[\mathrm{mJy} / \mathrm{beam}]}\end{array}$ & $\begin{array}{c}b_{\text {maj }} \\
{[\mathrm{mas}]}\end{array}$ & $\begin{array}{c}b_{\text {min }} \\
{[\mathrm{mas}]}\end{array}$ & $\begin{array}{c}\text { PA } \\
{\left[{ }^{\circ}\right]}\end{array}$ \\
\hline Natural & - & $624 \pm 94$ & $414 \pm 62$ & 0.99 & 0.407 & 0.075 & -10.1 \\
Natural (tapered) & 700 & $612 \pm 92$ & $412 \pm 62$ & 1.66 & 0.587 & 0.324 & -24.75 \\
Uniform & - & $565 \pm 85$ & $411 \pm 62$ & 1.21 & 0.354 & 0.057 & -9.3 \\
\hline
\end{tabular}

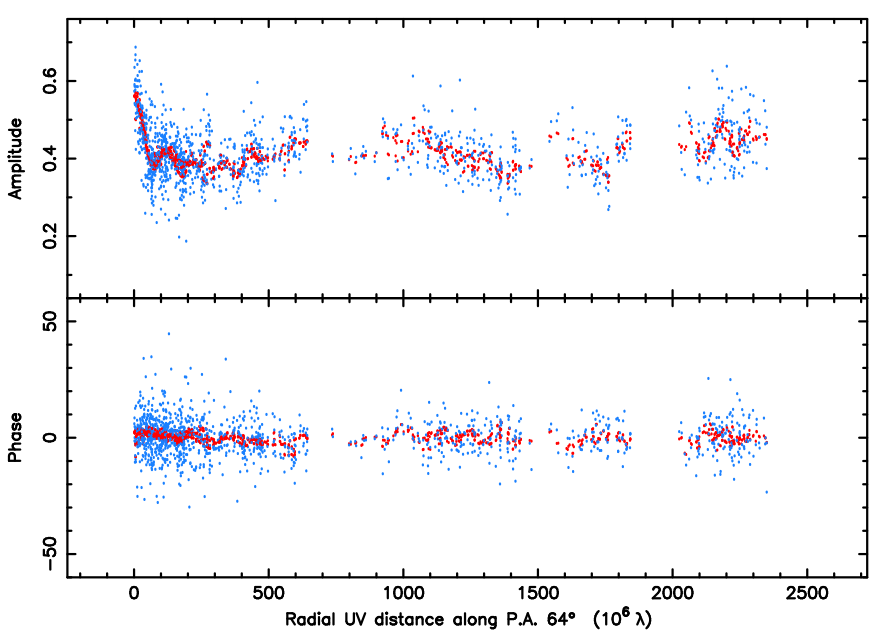

Fig. 2. Visibility amplitude (top panel) and phase (lower panel) vs. $(u, v)$-distance of NGC 1052 at $86 \mathrm{GHz}$ with the GMVA. The visibility data shown are uniformly weighted, post-self-calibrated, averaged per scan (240s) and projected along the jet positional angel of $64^{\circ}$. The blue and red dots correspond to the visibility data and clean model, respectively.

observation time. The process was repeated for subsequently smaller solution intervals of amplitude self-calibration.

We applied uniform weighting of the visibilities to achieve the best angular resolution. The imaging process was repeated several times to check for ambiguities in the final CLEAN model. Based on this and the amplitude of calibration factors, we estimate a conservative error of $15 \%$ on the flux densities in the final image. The image parameters are listed in Table 2. Figure 2 shows the visibility amplitude and phase versus radial $(u, v)$-distance in blue with the final clean model overplotted in red.

\section{Results}

The twin-jet system of NGC 1052 at the highest-angular resolution observed so far for this source is shown in Figs. 3 and 4 for uniform and natural weighting, respectively. It represents the highest linear-resolution image of an AGN ever taken. The minor axis of the Gaussian image restoring beam, which is oriented close to the east-west jet axis, is only $57 \mu$ as in diameter, which corresponds to $0.006 \mathrm{pc}$ or 7 light days. The image reveals a remarkably symmetric structure with one central feature at high brightness temperature and two fainter jets emanating from there to the east and west. A tapered map is shown in Fig. 4, giving a better impression on the overall structure. The two jets are partially resolved by the interferometric array and show a shallow brightness gradient out to about 2 mas from the center. Owing to the lack of short baselines, some diffuse flux may have been lost. More than $70 \%$ of the total correlated flux density is contained inside the central feature, which can be identified with

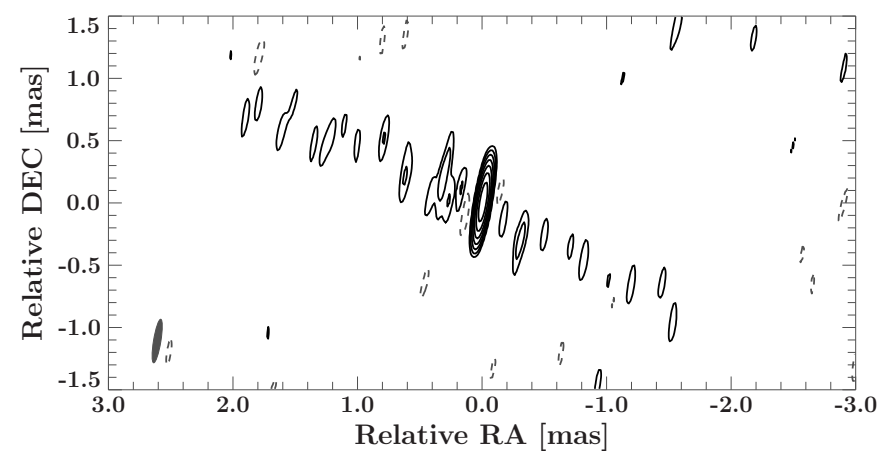

Fig. 3. Uniformly weighted GMVA image at $86 \mathrm{GHz}$ of the innermost structure of the bipolar jets in NGC 1052. Contour lines begin at three times the noise level and increase logarithmically by factors of 2. Image parameters are given in Table 2.
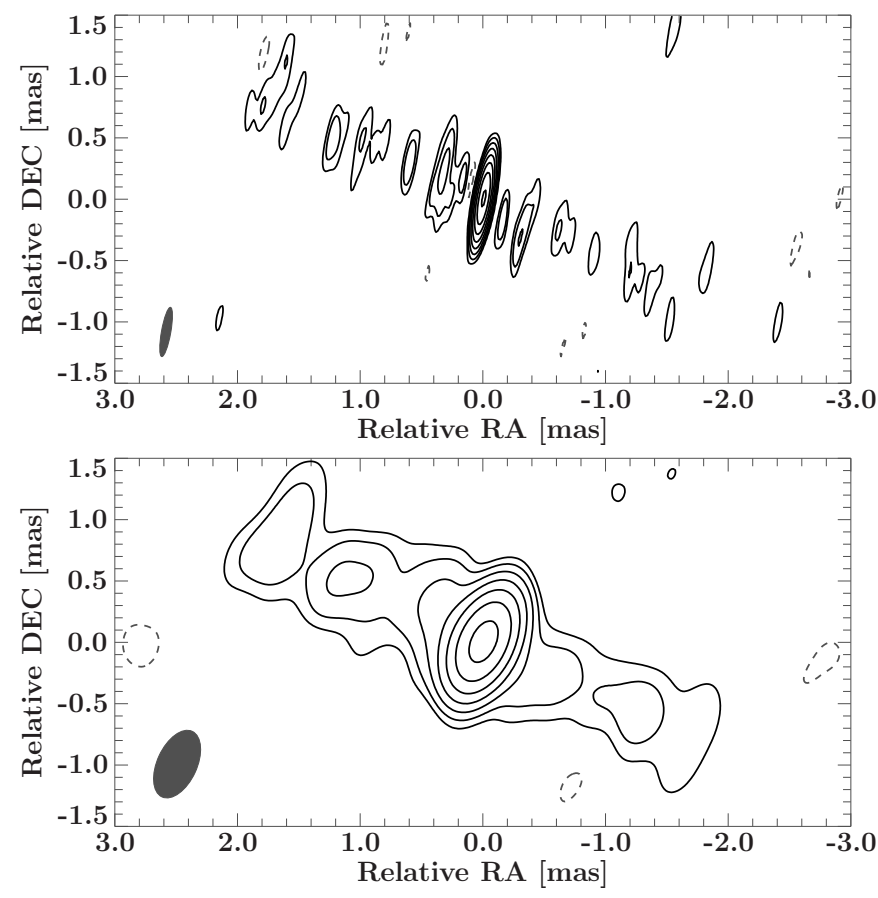

Fig. 4. Top: naturally weighted GMVA image at $86 \mathrm{GHz}$ of the innermost structure of the bipolar jets in NGC 1052; Bottom: with taper at $700 \mathrm{M} \lambda$. Contour lines begin at three times the noise level and increase logarithmically by factors of 2 . Image parameters are given in Table 2.

component B/B2 studied in earlier works (Kameno et al. 2001; Kadler et al. 2004a). Lower frequency studies suggested that the black hole is located $\sim(0.25$ to 0.50$)$ mas eastward of this region (Kadler et al. 2004a), thus ascribing the component to the western jet, which was assumed to be the counter jet (Kameno et al. 2001; Vermeulen et al. 2003; Kadler et al. 2004a). The striking 
symmetry in our new higher frequency and higher resolution image strongly suggests the true center of activity to be located inside the central feature, which then represents the unresolved emission of both jet cores and pinpoints the position of the supermassive black hole. We refer to the unresolved central feature as the $3 \mathrm{~mm}$ core of NGC 1052. This is one of the most precisely known locations of an extragalactic supermassive black hole in the Universe. Estimates have been obtained, for example, by Hada et al. (2011) for M87, by Fromm et al. (2013) for CTA 102, and Müller et al. (2014) for Centaurus A, although these jets are seen at a smaller angle to the line of sight, which leads to projection effects.

To further study the flux density distribution, the structure is divided into three parts, i.e., a center, a northeastern (NE) and a southwestern (SW) jet region. We summed up the CLEAN components within each region, based on the naturally weighted image, to gain a total flux density. All CLEAN components within 0.1 mas from the origin were attributed to the center. This yields a flux density for the NE and SW jet of $(118 \pm 18) \mathrm{mJy}$ and $(84 \pm 13) \mathrm{mJy}$, respectively. These components were localized well and separated from other jet components. The center contains $(415 \pm 62) \mathrm{mJy}(\sim 67 \%)$ of the total flux density in the image. We determine the position angle $\phi$ of both jets from the distribution of CLEAN components, yielding a weighted mean of $\left\langle\phi_{\mathrm{NE}}\right\rangle=(64 \pm 12)^{\circ}$ and $\left\langle\phi_{\mathrm{SW}}\right\rangle=(-120 \pm 13)^{\circ}$ for the $\mathrm{NE}$ and SW region, respectively, as measured from the north, with the errors representing the standard deviation. The CLEAN components were weighted with their flux density. These values are consistent with the median of $\phi$, i.e., $\phi_{\mathrm{NE}, \text { median }}=66^{\circ}$ and $\phi_{\text {SW,median }}=-118^{\circ}$. These values are consistent with previous works (Jones et al. 1984; Kadler et al. 2004b).

Our image marks the first time the two jets of NGC 1052 are detected by VLBI observations at $86 \mathrm{GHz}$. A previous $86 \mathrm{GHz}$ snapshot observation obtained by (Lee et al. 2008) with the Coordinated mm-VLBI Array in 2002 depicted only a single bright and compact feature of NGC 1052, most likely because of the lack of sensitivity to detect the jet. Our full-time GMVA observation achieved a dynamic range of 650:1, which is remarkable for such a low-luminosity and low-declination source.

The $3 \mathrm{~mm}$ core appears unresolved in the transverse (northsouth) direction (i.e., $\lesssim 150 \mu$ as, which corresponds to $\lesssim 1000 R_{\mathrm{S}}$ for a black hole mass of $10^{8.2} M_{\odot}$ ). The width of both jets can be measured, however, at a distance of about 0.2 mas east and west of the nucleus. We find a width of $\sim 0.2$ mas in both cases by fitting two Gaussian components at this distance. Thus, the opening angle of both jets has to be larger than $60^{\circ}$ within the inner 0.2 mas. This result is similar to the situation in the inner milliarcsecond of the M 87 core, which has been interpreted as a sign of a magnetohydrodynamically launched but still decollimated jet (Junor et al. 1999). In a region of such rapid expansion, possibly accompanied by differential expansion (Vlahakis \& Königl 2004), magnetic field energy can be converted efficiently into bulk kinetic energy, effectively accelerating the jets in this region. This result is in agreement with the abrupt change in brightness that we observe between the $86 \mathrm{GHz}$ $(3 \mathrm{~mm})$ core and the NGC 1052 jets.

\section{Discussion}

\subsection{Orientation of the twin-jet system}

To determine the orientation of the twin-jet system with respect to the line of sight, we measured the flux density in the CLEAN

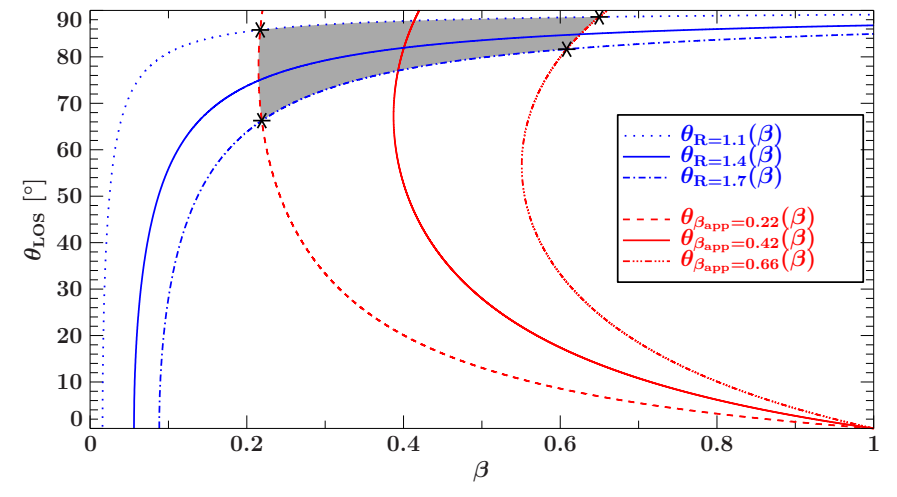

Fig. 5. Angle of the jet to the line of sight $\theta_{\mathrm{LOS}}$ depending on the jet velocity $\beta$ constrained by the jet-to-counter jet ratio Eq. (1)) measured at $86 \mathrm{GHz}$ (blue) and the apparent jet velocities (Eq. (2)) at $43 \mathrm{GHz}$, giving the mean, minimum, and maximum value (red). The allowed parameter space for $\theta_{\mathrm{LOS}}$ and $\beta$ is highlighted by the gray-shaded region.

model of our final image for the two jets separately, excluding the central feature from the regions used for the flux-density measurements of both jets (see Sect. 3). We thus found a fluxdensity ratio of $R=S_{\text {east }} / S_{\text {west }}=1.4 \pm 0.3$ and calculate the angle of the jet to the line of sight $\theta_{\text {jet }}$, which is linked to $R$ for a continuous jet via

$$
\frac{S_{\text {east }}}{S_{\text {west }}}=\left(\frac{1+\beta \cos \theta_{\mathrm{LOS}}}{1-\beta \cos \theta_{\mathrm{LOS}}}\right)^{2-\alpha},
$$

where $\beta$ is the intrinsic jet velocity and $\alpha$ the spectral index. Here, we use the measured spectral index of the jet $\alpha_{\text {jet }} \approx-1$ from lower frequencies (Kadler et al. 2004a). The inclination angle is also related to the intrinsic and apparent jet speeds $\beta$ and $\beta_{\text {app }}$ via

$$
\beta_{\mathrm{app}}=\frac{\beta \sin \theta_{\mathrm{LOS}}}{1-\beta \cos \theta_{\mathrm{LOS}}} .
$$

Since $\beta_{\text {app }}$ has not been measured at $86 \mathrm{GHz}$ for NGC 1052, we adopt a range of $\beta_{\text {app,min }}=0.22$ to $\beta_{\text {app, } \max }=0.66$ from a kinematic analysis at $43 \mathrm{GHz}$ over four years of VLBA observations (Baczko et al., in prep., see also Baczko 2015 and Baczko et al. 2015). This assumes a conservative range of jet speeds, which includes values from previous studies at lower frequencies as well (Vermeulen et al. 2003; Lister et al. 2013). However, Böck (2012) reported a maximum velocity of $\beta_{\text {app }}=0.42$ that is close to the averaged speed of $\beta_{\text {app }}=0.46 \pm 0.05$ derived from the coeval $43 \mathrm{GHz}$ kinematic study (Baczko et al., in prep.). The allowed range of parameters is highlighted by the gray-shaded area in Fig. 5. This yields $64^{\circ} \lesssim \theta_{\text {LOS }} \lesssim 87^{\circ}$ and $0.21 \lesssim \beta \lesssim 0.64$. The range of $\theta_{\text {LOS }}$ values shows the western jet of NGC 1052 to be oriented closer to the line of sight at $86 \mathrm{GHz}$ than at lower frequencies.

The orientation of the twin-jet system in NGC 1052 has previously been estimated using $\mathrm{cm}$-wavelength multifrequency and multiepoch observations to lie in the range $67^{\circ}$ to $72^{\circ}$, i.e., where the eastern jet is approaching and the western jet receding. This discrepancy might be explained by jet curvature on the innermost scales or by a slight intrinsic asymmetry between the two jets. In fact, the overall structure in our $86 \mathrm{GHz}$ GMVA image is similar to that shown at $43 \mathrm{GHz}$ in July 1997 (Vermeulen et al. 2003) but substantially more symmetric than in the $43 \mathrm{GHz}$ image of December 1998 (Kadler et al. 2004a). In fact, the substantial difference in the brightness ratio of the 
Table 3. Best core model-fit parameters for uniform weighting.

\begin{tabular}{lccccc}
\hline \hline Method & $\begin{array}{c}S_{\text {comp }} \\
{[\mathrm{mJy}]}\end{array}$ & $\begin{array}{c}a_{\text {maj }} \\
{[\mu \mathrm{as}]}\end{array}$ & $\begin{array}{c}a_{\text {min }} \\
{[\mu \mathrm{as}]}\end{array}$ & $\begin{array}{c}\text { PA } \\
{\left[{ }^{\circ}\right]}\end{array}$ & $\begin{array}{c}T_{\mathrm{b}} \\
{[\mathrm{K}]}\end{array}$ \\
\hline Elliptic modelfit & $409_{-2 \text { stat }}^{+3} \pm 62_{\text {sys }}$ & $14_{-14}^{+25}$ & 0 & -2.5 & - \\
Circular modelfit & $409_{-2 \text { stat }}^{+2} \pm 62_{\text {sys }}$ & $\leq 5$ & - & - & $\geq 3 \times 10^{12}$ \\
Lobanov (2005) & $409_{-2 \text { stat }}^{+3} \pm 62_{\text {sys }}$ & $\leq 39$ & $\leq 8$ & -2.5 & $\geq 2 \times 10^{11}$ \\
$1 / 5$ th beam & $409_{-2 \text { stat }}^{+3} \pm 62_{\text {sys }}$ & $\leq 57$ & $\leq 12$ & -2.5 & $\geq 1 \times 10^{11}$ \\
$1 / 2$ th beam & $409_{-2 \text { stat }}^{+3} \pm 62_{\text {sys }}$ & $\leq 88$ & $\leq 39$ & -2.5 & $\geq 1 \times 10^{11}$ \\
\hline
\end{tabular}

Notes. Uncertainties of the component parameters are given at the $1 \sigma$ level for one interesting parameter.

two jets between these two $43 \mathrm{GHz}$ VLBA observations indicates a possible intermittent and asymmetric injection of new plasma at the bases of both jets, which is also seen in a series of $43 \mathrm{GHz}$ VLBA observations in 2005/2006. We will investigate this further in a later publication. This, along with a possible underestimation of the strength of free-free absorption of the components at distances between 3 mas and 5 mas down the two jets (Kadler et al. 2004a) might have contributed to an underestimation of the inclination angle of the twin-jet system. All measurements of the inclination angle agree in the sense that the twin-jet system must be oriented close to the plane of the sky. As asymmetries are observed at lower frequencies, we cannot claim stability at $86 \mathrm{GHz}$, too. Our results represent the first snapshot observation at this frequency for NGC 1052, revealing the double-sided jet system. Therefore future observations at the same frequency are needed to give estimates on the stability of the jet system at $86 \mathrm{GHz}$.

\subsection{The core region: modeling the central emission feature}

To estimate the size of the emission region we fit the central feature in the uniformly weighted $86 \mathrm{GHz}$ GMVA image with an elliptical Gaussian component by replacing all CLEAN components within 0.05 mas of the image center. The fit was performed with ISIS (Houck \& Denicola 2000) with a direct connection to DifMAP (Shepherd et al. 1994). This allowed us to determine statistical errors on the fit parameter based on $\chi^{2}$ statistics (Grossberger 2014). Table 3 lists the resulting fit parameters with the statistical error given at the $1 \sigma$ level. In the uniformly weighted image we tested whether the component is unresolved, based on the $\mathrm{S} / \mathrm{N}$. We calculate the resolution limit $\theta_{\lim , \psi}$ as

$\theta_{\lim , \psi}=2^{2-(\beta / 2)}\left[\frac{\ln 2}{\pi} \ln \left(\frac{S / N}{S / N-1}\right)\right]^{1 / 2} b_{\psi}$,

where $\beta=0$, due to uniform weighting, $S / N=187$ is the ratio of the peak flux density and the noise level at the position of the component and $b_{\psi}$ is the beam axis at the position angle $\psi$ of the component (Lobanov 2005). The calculated limits for the minor and major axis of the component are larger than the fit, thus the elliptical Gaussian component is unresolved. The limit leads to an upper limit on the size of the emitting region along the jets of $<8 \mu$ as. A widely used empirical resolution limit is given by one-fifth of the beam size, which yields an upper limit on the size of $<12 \mu \mathrm{as}$. This estimate is slightly larger than that derived using Eq. (3). We applied a third approach for deriving the size of the central region by fitting two point sources in the direction of the jets. From an initial separation of $0.1 \mu$ as, we enlarged their distance until major changes in the residuals were observed. This was the case for a component separation of $\sim 30 \mu \mathrm{as}$, which is close to half the beam size. Thus using half the beam size would give the most conservative upper limit on the size of the emission region. The central feature is clearly unresolved, however, and having the power of super-resolution in VLBI observations (Martí-Vidal et al. 2012b) in mind, a smaller size is likely for our $3 \mathrm{~mm}$ observation. All derived values are listed in Table 3 .

The higher sensitivity that is to be expected from near-future VLBI arrays may improve the over-resolution power in the observations far above the diffraction limit currently achieved (Martí-Vidal et al. 2012b), thus leading to an even better modeling of the central region of NGC 1052.

\subsection{Magnetic field estimate}

\subsubsection{Radio emission mechanisms and location}

High-energy electrons in a strong magnetic field near the base of an AGN jet are accelerated and emit radio synchrotron radiation. The emitting electrons lose their energy quickly as they radiate. If the emitting particles change direction out of our line of sight or are not reaccelerated efficiently enough, the jet is not visible at this frequency beyond the point at which those particles have lost most of their energy. The $86 \mathrm{GHz}$ image of the jets in NGC 1052 shows a steep brightness drop from the core brightness to the much lower jet brightness within the first $15 \mu$ as or less.

It is also possible that the central emission could originate at least in part from the accretion disk. Models for accretion disks indicate that the emission can reach similar brightness temperatures as in our case, but these models are strongly parameter dependent (Asada et al. 2009). A detailed investigation of possible accretion disk models for NGC 1052 is beyond the scope of this paper. Hence, we consider the case with the fewest assumptions, i.e., that the flux density of the central region stems completely from the jet. Interpreting the central feature in this way makes it possible to derive the magnetic field in this area via synchrotron losses, assuming that the $86 \mathrm{GHz}$ emission disappears owing to synchrotron cooling.

\subsubsection{Estimating the magnetic field inside the $86 \mathrm{GHz}$ and $22 \mathrm{GHz}$ cores}

The magnetic field strength in the emitting region can be estimated with the following line of argument (Rybicki \& Lightman 2007; Böttcher 2012). The synchrotron cooling time of a particle 
radiating at $86 \mathrm{GHz}$ is given by the Larmor formula for relativistic particles. Assuming a random pitch angle distribution, the emitted power for an electron is

$P=\frac{4}{3} c \sigma_{\mathrm{T}} u_{B} \beta^{2} \gamma^{2}$,

where $\gamma$ is the Lorentz factor and $\beta$ the ratio of the speed of the radiating electrons to the speed of light, $\sigma_{\mathrm{T}}$ the Thomson cross section, and $u_{B}$ the energy density of the magnetic field.

We can express the energy loss of the electron due to radiation in terms of the change of its Lorentz factor

$\left(\frac{\mathrm{d} \gamma}{\mathrm{d} t}\right)=-\frac{4}{3} \sigma_{\mathrm{T}} \frac{u_{B}}{m_{\mathrm{e}} \cdot c} \gamma^{2} \beta^{2}$

where $m_{\mathrm{e}}$ is the electron mass. Integration yields the cooling time, dependent on the energy and radiation rate of the electron, for a given Lorentz factor

$t_{\mathrm{c}}=\frac{3}{4} \frac{m_{\mathrm{e}} c}{\gamma \sigma_{\mathrm{T}} u_{B} \beta^{2}}$.

Inserting the energy density of the magnetic field and the critical frequency (frequency at which the spectral output peaks),

$v_{\mathrm{c}} \equiv \frac{3}{4 \pi} \frac{e B}{m_{\mathrm{e}} c} \gamma^{2}$,

where $e$ is the charge of the electron and $B$ is the magnetic field, the cooling time becomes

$t_{\mathrm{c}}=3 \sqrt{3 \pi} \sqrt{\frac{m_{\mathrm{e}} c e}{v_{\mathrm{c}}}} \frac{1}{\sigma_{\mathrm{T}} \beta^{2}} B^{-3 / 2}=5.4 \times 10^{6} B^{-3 / 2} /\left[\mathrm{G}^{-2 / 3}\right][\mathrm{s}]$,

inserting the Thomson cross-section $\sigma_{\mathrm{T}}=6.65 \times 10^{-25} \mathrm{~cm}^{2}$, the electric charge $e=4.8 \times 10^{-10} \mathrm{C}$, the mass of the electron $m_{\mathrm{e}}=$ $9.11 \times 10^{-28} \mathrm{~g}$, and a critical frequency of $v_{\mathrm{c}} \simeq 86 \mathrm{GHz}$.

As the emitting particles are advected with the flow, this expression can be related to the size of the core by means of the bulk speed of the jet.

Setting the travel distance of the electrons during this time equal to the assumed resolution limit, we obtain for the case of synchrotron cooling

$B_{\mathrm{sc}, d}=\left(\frac{d /[\mathrm{cm}]}{\beta\left[\mathrm{cm} \mathrm{s}^{-1}\right] \times 5.4 \times 10^{6} \mathrm{~s}}\right)^{-2 / 3}[\mathrm{G}]$,

which depends on the bulk speed $\beta$ of the jet and the size $d$ of the emitting region. This gives the mean magnetic field intensity of the region from which the radiation is emitted.

Therefore, the smallest magnetic field estimate follows from the most conservative resolution limit, which leads to a size along the jet of $\lesssim 30 \mu$ as $\left(\sim 0.003 \mathrm{pc}\right.$ or $\left.\sim 200 R_{\mathrm{S}}\right)$. Interpreting this region as blended emission from both jet bases, this size constrains the distance of the central engine and the base of the $3 \mathrm{~mm}$ core to be $\lesssim 100 R_{\mathrm{S}}$ (see Fig. 6). The largest estimate of the magnetic field strength results from the smallest possible size of the emission region of $4 R_{\mathrm{S}}(0.6 \mu$ as or $0.06 \mathrm{mpc})$, which is likely to be an underestimation.

As discussed in section 4.1. a kinematic study at $43 \mathrm{GHz}$ revealed a range of $0.22 \leq \beta_{\text {app }} \leq 0.66$, resulting in an averaged bulk speed of $\beta_{\text {app }}=0.46 \pm 0.05$ for the overall jet flow (see also Baczko 2015; and Baczko et al. 2015). Both the bulk speed and the cooling distance have the same weight in deriving $B_{\mathrm{sc}, \mathrm{d}}$
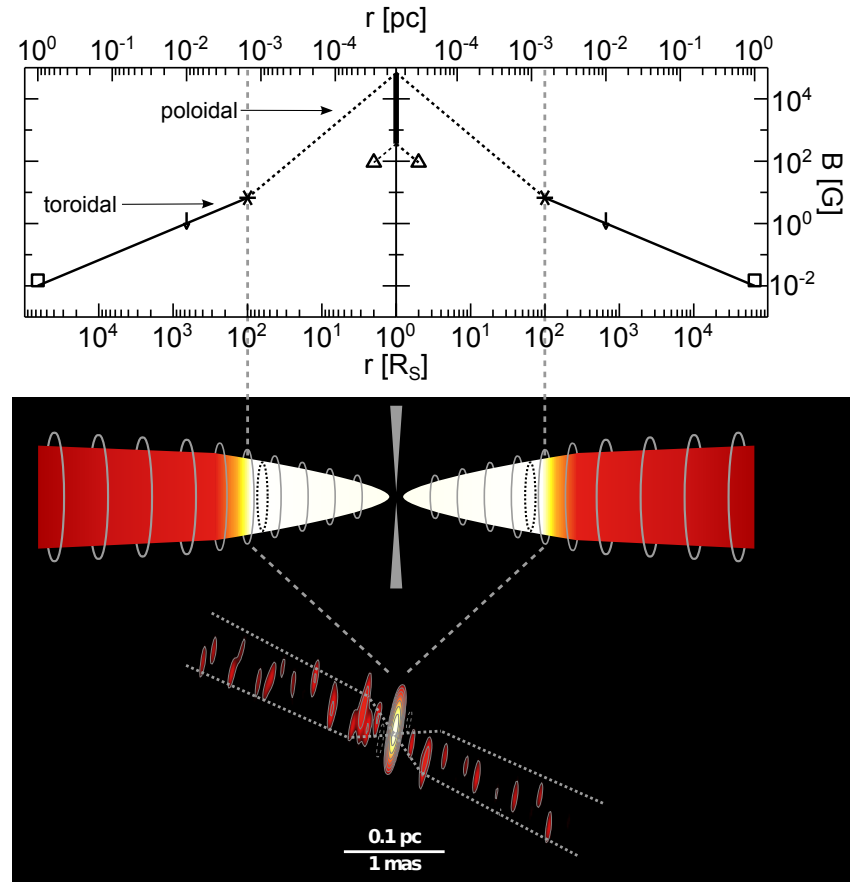

Fig. 6. Sketch of the jet-launching region. The mm VLBI image (bottom panel) resolves structures larger than $\sim 100$ to 400 Schwarzschild radii $\left(R_{\mathrm{S}}\right)$ around the central supermassive black hole. In the innermost region, both jets have extremely large opening angles of $>60^{\circ}$. The regions at which the two jets become optically thick, usually referred to as the two jet cores (dashed ellipses in middle part), are located at even smaller scales $\left(<100 R_{\mathrm{S}}\right)$. The magnetic field (top panel) has a toroidal configuration $B \propto r^{-1}$ (solid line). Near the black hole, a poloidal magnetic field may start to dominate (dashed lines). The asterisk and triangle show the measured magnetic field from synchrotron cooling, using the most conservative and the lowest possible estimate of the size of the central region, respectively. From the former the toroidal field out to $1 \mathrm{pc}$ and the poloidal field down to $1 R_{\mathrm{S}}$ are extrapolated, while from the latter only the poloidal field down to $1 R_{\mathrm{S}}$ is extrapolated. The thick line in the middle of the upper panel indicates the allowed range for the magnetic field at $1 R_{\mathrm{S}}$. The downward arrow and square indicate the upper limit from SSA and core-shift measurements, respectively.

(compare Eq. (9)), however, the large uncertainty in the cooling distance makes its impact in Eq. (9) larger. Therefore, the following discussion first focuses on the different estimates on the size of the central region and assumes the averaged speed resulting from the $43 \mathrm{GHz}$ analysis. Taking the projection effects into account (compare Sect. 4.1), the changes are negligible in the scope of the herein assumed accuracy, resulting in $\beta \simeq 0.5$ for this case. After deriving the magnetic field at different regions of the jets, we briefly discuss the impact of the full range of velocities (from the minimal to the maximal measured velocities) on our results.

Assuming a bulk speed of $\beta \simeq 0.5$ and the cooling distance ranging from $0.3 \mu$ as to $15 \mu \mathrm{as}$, Eq. (9) provides a range of $6.7 \mathrm{G}$ to $91 \mathrm{G}$ for the magnetic field at the edge the central region. Another independent approach gives an upper limit on the magnetic field at $0.01 \mathrm{pc}(0.1 \mathrm{mas})$ distance from the black hole. This can be derived from the synchrotron self absorption (SSA) spectrum of the central component. The peak frequency of the SSA, assuming equipartition between the magnetic field and the nonthermal electrons is

$v_{\mathrm{m}} \sim 3.2 B^{1 / 5} /\left[\mathrm{G}^{1 / 5}\right] S_{m} /[\mathrm{Jy}] \theta^{-4 / 5} /\left[\mathrm{mas}^{-4 / 5}\right](1+z)^{1 / 5}[\mathrm{GHz}]$, 
Table 4. Magnetic field values.

\begin{tabular}{|c|c|c|c|c|c|c|}
\hline Method & $\begin{array}{l}\text { Region size } d \\
{[\mu \text { as }]}\end{array}$ & $\begin{array}{l}\text { Region size } d \\
{[\mathrm{mpc}]}\end{array}$ & $\begin{array}{l}B_{\mathrm{sc} d} \\
{[\mathrm{G}]}\end{array}$ & $\begin{array}{c}B_{\mathrm{sc}, 1 \mathrm{pc}} \\
{[\mathrm{G}]}\end{array}$ & $\begin{array}{c}B_{\mathrm{Sc}, 1 R_{\mathrm{s}}}{ }^{a} \\
\quad[\mathrm{G}]\end{array}$ & $\begin{array}{c}B_{\mathrm{sc}, 1 R_{\mathrm{s}}}{ }^{2} \\
\quad[\mathrm{G}]\end{array}$ \\
\hline $4 R_{\mathrm{S}}$ & 0.6 & 0.06 & $\geq 91$ & $\geq 0.003$ & $\simeq 360$ & $\simeq 360$ \\
\hline Lobanov (2005) & 8 & 0.8 & $\geq 16$ & $\geq 0.007$ & $\geq 880$ & $\leq 1.3 \times 10^{4}$ \\
\hline $1 / 5$ th beam $^{c}$ & 12 & 1.2 & $\geq 12$ & $\geq 0.008$ & $\geq 1000$ & $\leq 2.1 \times 10^{4}$ \\
\hline Half a beam ${ }^{c}$ & 30 & 3.1 & $\geq 6.7$ & $\geq 0.01$ & $\geq 1400$ & $\leq 6.9 \times 10^{4}$ \\
\hline
\end{tabular}

Notes. ${ }^{(a)}$ Assuming $B \propto r^{-1}$ for $r>2 R_{\mathrm{S}}$ and $B \propto r^{-2}$ for $r<2 R_{\mathrm{S}} ;^{(b)} B \propto r^{-2}$ for $r<d$; ${ }^{(c)}$ beam at $86 \mathrm{GHz}$, value used is FWHM of the beam at position angle $64^{\circ}$ along the jet axis.

resulting in units of $\mathrm{GHz}$ for $v_{m} . \theta$ is the angular size of the source (Kellermann \& Pauliny-Toth 1981), $z=0.005$ is the redshift, and $S_{m}$ is the maximum value of the flux density. Using the brightness temperature $T_{\mathrm{b}}$ of a given component, the magnetic field strength can be thus derived as

$B_{\mathrm{SSA}} \sim 4.57 \times 10^{19} v_{m} /[\mathrm{GHz}] T_{\mathrm{b}}^{-2} /\left[\mathrm{K}^{-2}\right](1+z)[\mathrm{G}]$.

The spectrum of the central core component peaks at $\simeq 22 \mathrm{GHz}$ (Kadler et al. 2004a). As free-free absorption also takes place, this gives us an upper limit on the SSA peak frequency $v_{\mathrm{m}}$. The innermost component $\mathrm{B} 2 \mathrm{~b}$ has $T_{\mathrm{b}} \simeq 2.0 \times 10^{10} \mathrm{~K}$ (Kadler et al. 2004a). Converting $T_{\mathrm{b}}$ and $v_{\mathrm{m}}$ to the jet rest frame (using a viewing angle of $86^{\circ}$, spectral index $\alpha=0.0$, and flow speed $\beta=0.25)$ gives $T_{\mathrm{b}}^{*}=2.2 \times 10^{10} \mathrm{~K}$ and $v_{\mathrm{m}}^{*}=23 \mathrm{GHz}$. Adopting a core size of $(0.21 \pm 0.01)$ mas leads to $B_{\mathrm{SSA}}<2.5 \mathrm{G}$. This value can be thus considered as an upper limit on the magnetic field intensity at a distance of $0.01 \mathrm{pc}(0.1 \mathrm{mas})$ along the jet. This upper limit is in agreement with the expectations for a toroidal magnetic field decreasing as $r^{-1}$ between the $3 \mathrm{~mm}$ core and 0.1 mas $(1 \mathrm{G}$ would be expected in this scenario for the largest black hole $3 \mathrm{~mm}$ core distance of $0.0015 \mathrm{pc}$ ), assuming that the jet is adiabatically expanding.

A third independent estimate of the magnetic field in the jet is given by the measurement of the core-shift effect (Zamaninasab et al. 2014), assuming equipartitioning of the energy between the magnetic field and the nonthermal particles. This approach yields a magnetic field of $15 \mathrm{mG}$ at $1 \mathrm{pc}$ (10 mas). This is in remarkable agreement with the expectation for a $r^{-1}$ decrease of the $6.7 \mathrm{G}$ field from $0.0015 \mathrm{pc}$ out to $1 \mathrm{pc}$ for which $10 \mathrm{mG}$ would be expected. Together, these three estimates of the magnetic field along the twin jets of NGC 1052 yield a self-consistent picture of a toroidal magnetic field dominating outside the $3 \mathrm{~mm}$ core and decreasing outward proportional to $r^{-1}$. So far, an $r^{-1}$ dependence has been adopted in the literature to estimate the inner magnetic field close to the black hole based on plausibility (O'Sullivan \& Gabuzda 2009; Pushkarev et al. 2012).

The gradient of the toroidal magnetic field is thus measured fully on scales from the $3 \mathrm{~mm}$ core to $1 \mathrm{pc}$ and may be extrapolated further inward as a lower limit. At some distance closer than $0.0015 \mathrm{pc}$, near the central engine, a poloidal magnetic field with a $r^{-2}$ dependence is expected to start dominating over the toroidal field (Komissarov 2012), that is defined by the spin parameter, which we assumed as $a=1$ for the case of a probably rapidly rotating black hole in NGC 1052 (Brenneman et al. 2009). If effective magnetic acceleration of the jet takes place, converting magnetic into bulk kinetic energy, this gradient might steepen considerably.

We assume a toroidal magnetic field from the $3 \mathrm{~mm}$ core until $2 R_{\mathrm{S}}$, where the poloidal field starts to dominate (Komissarov 2012). This leads to a dependence of the magnetic field at $1 R_{\mathrm{S}}$ on the size of the emitting region $d$ of $B_{\mathrm{sc}, 1 \mathrm{R}_{\mathrm{S}}} \propto d^{1 / 3}$. Assuming an unrealistically small size for this region of $4 R_{\mathrm{S}}$ gives the lowest possible magnetic field strength at $1 R_{\mathrm{S}}$ of $\simeq 360 \mathrm{G}$. At these scales one should check whether the temporal shift due to the central mass should be taken into account. The scaling factor of 1.15 is negligible in the scope of the uncertainty of the magnetic field. The maximum upper limit on the emission region that is still in agreement with our analysis is half of the beam. Assuming a poloidal magnetic field from this distance toward the central engine gives an upper limit on the magnetic field at $1 R_{\mathrm{S}}$ of $\leq 6.9 \times 10^{4} \mathrm{G}$. An overview of all the magnetic field values derived in the previous paragraphs is given in Table 4.

The range of the magnetic field follows by assuming an averaged bulk speed of $\beta_{\text {app }}=0.5$. As discussed in Sect. 4.1 it is the highest averaged value until now, but not far away from other estimates. For example assuming a velocity of $\beta_{\text {app }}=0.4$ (maximum values reported by Lister et al. 2013, Böck 2012) gives only slight deviations, leading to $310 \mathrm{G} \leq B_{\mathrm{sc}, 1 R_{\mathrm{S}}} \leq 6.0 \times 10^{4} \mathrm{G}$. Magnetic fields with boundaries from $200 \mathrm{G}$ to $430 \mathrm{G}$, and $4.0 \times$ $10^{4} \mathrm{G}$ to $8.3 \times 10^{4} \mathrm{G}$ are implied, following the highest and lowest velocity values measured at $43 \mathrm{GHz}$ (Baczko et al., in prep., see also Baczko 2015 - compare Fig. 5). Therefore, the impact of the uncertainty in the apparent bulk speed on the magnetic field strength is negligible compared to that resulting from the size of the central region. This is probably caused by the larger uncertainty in the region size compared to the velocity estimates on the jet flow.

An important target with which to compare our results is M 87. It is located at a distance of 16.7 Mpc (Jordán et al. 2005) similar to NGC 1052. The mass of the central black hole in M 87 $\left(6 \times 10^{9} M_{\odot}\right.$ Gebhardt \& Thomas 2009) is about two orders of magnitude larger than in NGC 1052, hence, the Schwarzschild radius of the central black hole in M 87 is about two orders of magnitude larger than that of NGC 1052. This is, however, partially countered by the difference in inclination angle to the line of sight. At a $15^{\circ}$ to $25^{\circ}$ inclination angle (Acciari et al. 2009; Biretta et al. 1999), all linear scales in the M 87 jet are affected by a projection with a factor of 0.26 to 0.42 . This results in $\sim 7 \mu$ as corresponding to $1 R_{\mathrm{S}}$ in M 87 . Thus, formally, VLBI observations can probe structures that are about 30 times smaller in units of Schwarzschild radii in M 87 than in NGC 1052. Two additional effects play in favor of NGC 1052: I) distances along the two-jet system can be measured with double precision, if intrinsic symmetry between the two jets is assumed, and II) the orientation of the projected jet system is nearly perfect in the eastwest direction and is conveniently aligned well with the minimal width of the elliptical restoring beam for northern VLBI arrays to give us the best resolution along the jet axis.

Based on core-shift measurements, the magnetic field inside the $43 \mathrm{GHz}$ jet core of M 87 has been measured (Kino et al. 2014) to lie in the range ( 1 to 15 ) $\mathrm{G}$ (at a projected distance 
of $\sim 6 R_{\mathrm{S}}$ to the jet base). Based on their analysis Kino et al (2014) excluded magnetic fields as strong as $\left(10^{3}\right.$ to $\left.10^{4}\right) \mathrm{G}$. These are usually assumed to explain the formation of AGN jets via extraction of magnetic energy from the rotational energy of the black hole via the Blandford \& Znajek (1977) mechanism and has been estimated for blazars (O'Sullivan \& Gabuzda 2009; Pushkarev et al. 2012). These authors showed that the jet base in M 87 is located within (14 to 23$) R_{\mathrm{S}}$ of its radio core at $43 \mathrm{GHz}$ (Hada et al. 2011). At frequencies of $228 \mathrm{GHz}$, corresponding to $1.3 \mathrm{~mm}$ wavelength, the size of the $\mathrm{M} 87$ jet core has been measured to be as small as $5.5 R_{\mathrm{S}}$. This small size is comparable to the predicted inner edge of a corotating accretion disk around a Kerr black hole (Doeleman et al. 2012). Based on this observation Kino et al. (2015) derived the magnetic field inside the $228 \mathrm{GHz}$ core to $58 \mathrm{G} \leq B_{\text {tot }} \leq 127 \mathrm{G}$ for a region size of $21 \mu$ as $\leq \theta \leq 25.5 \mu$ as. Extrapolating the result from core shift measurements (Hada et al. 2011) down to this frequency, the $228 \mathrm{GHz}$ core is located at a deprojected distance of (2.5 to 4) $R_{\mathrm{S}}$ (Doeleman et al. 2012). Therefore the magnetic field estimate is in agreement with the lower edge of our magnetic field derivation inside the $86 \mathrm{GHz}$ core $(\sim 90 \mathrm{G}$ for a distance from the black hole of $2 R_{\mathrm{S}}$ ). The jet shows a wide opening angle of $\sim 60^{\circ}$ in the innermost region and is collimated within $\sim 30$ to $100 R_{\mathrm{S}}$ of the core (Junor et al. 1999). This provides circumstantial evidence that the $1.3 \mathrm{~mm}$ jet core is located very close to the black hole. This hypothesis cannot be confirmed by approaching the same location from the counter jet side as the flux density of the counter jet (Kovalev et al. 2007) in M 87 is too low and the angle to the line of sight (Acciari et al. 2009) is only (15 to 25) An additional offset between the black hole and the jet base, as well as a steep magnetic field gradient, might still increase the peak magnetic field strength near the black hole into the Blandford \& Znajek regime, but this hypothesis is difficult to test in more inclined sources with a high jet-to-counter jet brightness ratio like that in $\mathrm{M} 87$. An even higher magnetic field strength on scales of $0.01 \mathrm{pc}$ has been reported by Martí-Vidal et al. (2015) for PKS 1830-211, leading to tens of gauss on these scales.

Summarizing, we have obtained independent estimates of the magnetic field intensity at two different distances from the black hole $(0.5 \mathrm{mpc}$ and $10 \mathrm{mpc})$. We used basic synchrotron theory, which, together with a third estimate from core shift at $1 \mathrm{pc}$ (Zamaninasab et al. 2014), is consistent with the magnetic field intensity falling with $r^{-1}$, i.e., implying that the magnetic field structure is already dominated by the toroidal component at subparsec scales. The peak magnetic field in both scenarios exceeds the value of $\sim 200 \mathrm{G}$ needed to launch the jet with kinetic power of $5 \times 10^{41} \mathrm{erg} \mathrm{s}^{-1}$ (as derived by Kadler et al. 2003) via the Blandford \& Znajek mechanism (Blandford \& Znajek 1977; Komissarov 2012).

\section{Conclusion and summary}

Our analysis of the highest resolution VLBI observation of NGC 1052 at $86 \mathrm{GHz}$ with the GMVA from 2004-10-09 shows the twin jet of NGC 1052 extending from a single core without any indication of absorption due to the obscuring torus seen at lower frequencies. The average flux density ratio between both jets is determined to be $1.40 \pm 0.30$ (being the eastern jet brighter). In combination with measurements of the apparent jet velocity at lower frequencies, we determine the angle of the jet to the line of sight to be $\sim 86^{\circ}$. Instead of seeing two separate radio cores, the two jets extend from a single central region with a size of $<200 R_{\mathrm{S}}$. The center in our image of
NGC 1052 may include the radio core of each jet, contributing to the emission in this part. From an estimate of the synchrotron cooling time it was possible to derive a magnetic field at $1 R_{\mathrm{S}}$ of $360 \mathrm{G}<B<6.9 \times 10^{4} \mathrm{G}$. We have shown that it is feasible to reach sufficient sensitivity with VLBI at $86 \mathrm{GHz}$ to detect the two jets. A kinematic study and higher sensitivity observations at $86 \mathrm{GHz}$ are crucial to further constrain the jet geometry and compare the evolution of the jet with numerical models of bipolar jet production, especially in terms of asymmetries in the jet outflow. Adding space-based VLBI will improve the resolution in the north-south direction and going to even higher frequencies will improve the overall resolution. This makes NGC 1052 one of the very few, very close and bright targets to test the unification scheme of AGN and jet formation on scales of several light days in a twin-jet system.

Acknowledgements. We thank Alan Roy for his insightful comments, which improved the manuscript. We acknowledge support and partial funding by the Deutsche Forschungsgemeinschaft grant WI 1860-10/1 and GRK 1147, the Spanish MINECO project AYA2012-38491-C02-01, AYA2013-4079-P, and AYA2013-48226-C03-02-P, the Generalitat Valenciana projects PROMETEOII/2014/057, and PROMETEOII/2014/069, as well as by the COST MP0905 action "Black Holes in a Violent Universe". This research has made use of the software package ISIS (Houck \& Denicola 2000) and a collection of ISIS scripts provided by the Dr. Karl Remeis Observatory, Bamberg, Germany at http://www.sternwarte.uni-erlangen.de/isis/, as well as an ISISDIFMAP fitting setup by C. Grossberger (Grossberger 2014). This research has made use of the NASA/IPAC Extragalactic Database (NED) which is operated by the Jet Propulsion Laboratory, California Institute of Technology, under contract with the National Aeronautics and Space Administration. This research has made use of data obtained with the Global Millimeter VLBI Array (GMVA), which consists of telescopes operated by the MPIfR, IRAM, Onsala, Metsähovi, Yebes and the VLBA. The data were correlated at the correlator of the MPIfR in Bonn, Germany. The VLBA is an instrument of the National Radio Astronomy Observatory, a facility of the National Science Foundation operated under cooperative agreement by Associated Universities, Inc.

\section{References}

Acciari, V. A., Aliu, E., Arlen, T., et al. 2009, Science, 325, 444 Antonucci, R. 1993, ARA\&A, 31, 473

Asada, K., Doi, A., Kino, M., et al. 2009, in Approaching Micro-Arcsecond Resolution with VSOP-2: Astrophysics and Technologies, eds. Y. Hagiwara, E. Fomalont, M. Tsuboi, \& M. Yasuhiro, ASP Conf. Ser., 402, 262

Baczko, A.-K. 2015, Master's Thesis, Friedrich-Alexander-Universität Erlangen-Nürnberg

Baczko, A.-K., Schulz, R., Kadler, M., et al. 2015, Millimeter VLBI Observations of the Twin-Jet-System in NGC1052, in Dissecting the Universe, https://events.mpifr-bonn.mpg.de/indico/event/4/ session/1/contribution/5

Biretta, J. A., Sparks, W. B., \& Macchetto, F. 1999, ApJ, 520, 621

Blandford, R. D., \& Znajek, R. L. 1977, MNRAS, 179, 433

Böttcher, M., \& Anita, R. 2012, Radiation Processes, eds. M. Boettcher, D. E. Harris, \& H. Krawczynski (John Wiley and Sons), 39

Brenneman, L. W., Weaver, K. A., Kadler, M., et al. 2009, ApJ, 698, 528

Böck, M. 2012, Ph.D. Thesis, Friedrich-Alexander-Universität ErlangenNürnberg

Doeleman, S. S., Fish, V. L., Schenck, D. E., et al. 2012, Science, 338, 355

Fanidakis, N., Baugh, C. M., Benson, A. J., et al. 2011, MNRAS, 410, 53

Fromm, C. M., Ros, E., Perucho, M., et al. 2013, A\&A, 557, A105

Gebhardt, K., \& Thomas, J. 2009, ApJ, 700, 1690

Grossberger, C. 2014, Ph.D. Thesis, Friedrich-Alexander-Universität ErlangenNürnberg

Hada, K., Doi, A., Kino, M., et al. 2011, Nature, 477, 185

Houck, J. C., \& Denicola, L. A. 2000, in Astronomical Data Analysis Software and Systems IX, eds. N. Manset, C. Veillet, \& D. Crabtree, ASP Conf. Ser., 216,591

Jones, D. L., Wrobel, J. M., \& Shaffer, D. B. 1984, ApJ, 276, 480

Jordán, A., Côté, P., Blakeslee, J. P., et al. 2005, ApJ, 634, 1002

Junor, W., Biretta, J. A., \& Livio, M. 1999, Nature, 401, 891

Kadler, M., Ros, E., Kerp, J., et al. 2003, New Astron. Rev., 47, 569

Kadler, M., Kerp, J., Ros, E., et al. 2004a, A\&A, 420, 467 
Kadler, M., Ros, E., Lobanov, A. P., Falcke, H., \& Zensus, J. A. 2004b, A\&A, 426,481

Kameno, S., Sawada-Satoh, S., Inoue, M., Shen, Z.-Q., \& Wajima, K. 2001, PASJ, 53, 169

Kellermann, K. I., \& Pauliny-Toth, I. I. K. 1981, ARA\&A, 19, 373

Kino, M., Takahara, F., Hada, K., \& Doi, A. 2014, ApJ, 786, 5

Kino, M., Takahara, F., Hada, K., et al. 2015, ApJ, 803, 30

Komissarov, S. 2012, in Relativistic Jets from Active Galactic Nuclei, eds. M. Boettcher, D. E. Harris, \& H. Krawczynski (Wiley-VCH), 81

Kovalev, Y. Y., Lister, M. L., Homan, D. C., \& Kellermann, K. I. 2007, ApJ, 668 L27

Lee, S.-S., Lobanov, A. P., Krichbaum, T. P., et al. 2008, AJ, 136, 159

Lister, M. L., \& Marscher, A. P. 1997, ApJ, 476, 572

Lister, M. L., Cohen, M. H., Homan, D. C., et al. 2009, AJ, 138, 1874

Lister, M. L., Aller, M. F., Aller, H. D., et al. 2013, AJ, 146, 120

Lobanov, A. P. 2005, ArXiv e-prints [arXiv: astro-ph/0503225]

Martí-Vidal, I., Krichbaum, T. P., Marscher, A., et al. 2012a, A\&A, 542, A107
Martí-Vidal, I., Pérez-Torres, M. A., \& Lobanov, A. P. 2012b, A\&A, 541, A135

Martí-Vidal, I., Muller, S., Vlemmings, W., Horellou, C., \& Aalto, S. 2015, Science, 348,311

Müller, C., Kadler, M., Ojha, R., et al. 2014, A\&A, 569, A115

O'Sullivan, S. P., \& Gabuzda, D. C. 2009, MNRAS, 400, 26

Pushkarev, A. B., Hovatta, T., Kovalev, Y. Y., et al. 2012, A\&A, 545, A113

Rybicki, G. B., \& Lightman, A. P. 2007, Synchrotron Radiation (Weinheim, Germany: Wiley-VCH Verlag $\mathrm{GmbH}$ )

Shepherd, M. C., Pearson, T. J., \& Taylor, G. B. 1994, BAAS, 26, 987

Vermeulen, R. C., Ros, E., Kellermann, K. I., et al. 2003, A\&A, 401, 113

Vlahakis, N., \& Königl, A. 2004, ApJ, 605, 656

Woo, J.-H., \& Urry, C. M. 2002, ApJ, 579, 530

Wrobel, J. M. 1984, ApJ, 284, 531

Zamaninasab, M., Clausen-Brown, E., Savolainen, T., \& Tchekhovskoy, A 2014, Nature, 510, 126

Zensus, J. A. 1997, ARA\&A, 35, 607 